\title{
Respiratory impedance response to a deep inhalation in asthmatic children with spontaneous airway obstruction
}

\author{
C. Schweitzer, C. Moreau-Colson, F. Marchal
}

Respiratory impedance response to a deep inhalation in asthmatic children with spontaneous airway obstruction. C. Schweitzer, C. Moreau-Colson, F. Marchal. (C) ERS Journals Ltd 2002.

ABSTRACT: The aim of the study was to determine whether the bronchomotor effect of a deep inhalation (DI) may be detected during tidal breathing in asthmatic children with spontaneous airway obstruction (AO).

Two groups of children aged 5-15 yrs were studied. AO was mild in group $1(n=12$, forced expiratory volume in one second (FEV1) $\geqslant 75 \%$ predicted) and moderateto-severe in group $2(\mathrm{n}=9, \mathrm{FEV} 1 \leqslant 70 \%$ pred $)$. The forced oscillation technique at $12 \mathrm{~Hz}$ using a head generator allowed the determination of respiratory resistance in inspiration (Rrsi) and expiration (Rrse) before and after DI, at baseline and after salbutamol.

At baseline, $R$ rsi but not $R$ rse was found to decrease significantly after DI in group 1 but not in group 2 . The change induced by DI was significantly different in group 1 $\left(-1.5 \pm 0.5 \mathrm{hPa} \cdot \mathrm{s} \cdot \mathrm{L}^{-1}\right)$ compared to group $2\left(0.5 \pm 0.5 \mathrm{hPa} \cdot \mathrm{s} \cdot \mathrm{L}^{-1}\right)$ and exhibited significant negative correlation to $\mathrm{FEV} 1 \%$ pred. After salbutamol, DI had no effect.

In conclusion, asthmatic children show a bronchomotor response to deep inhalation that depends on the degree of airway obstruction. The effect is more readily demonstrated in inspiration than in expiration.

Eur Respir J 2002; 19: 1020-1025.
Laboratoire d'Explorations Fonctionnelles Pédiatriques, Hôpital d'Enfants, Centre Hospitalier Universitaire de Nancy, Vandoeuvre, France.

Correspondence: F. Marchal, Laboratoire de Physiologie, Faculté de Médecine, Avenue de la Forêt de Haye, F-54505, Vandoeuvre les Nancy, France.

Fax: 33383592726

E-mail: f.marchal@chu-nancy.fr

Keywords: Asthma, deep inhalation, forced oscillation technique, head generator, paediatric lung function, respiratory resistance and reactance

Received: July 62001

Accepted after revision January 122002

This study was supported by EA 3450 "Interactions des systèmes de régulation respiratoire chez l'adulte et l'enfant".
The effect of deep inhalation (DI) may be of particular relevance to the study of respiratory function in subjects with diseased airways, as it may provide indications relative to the mechanisms of airway obstruction (AO) $[1,2]$. The consequence of DI on airway mechanics appears to be determined mainly by the hysteresis of conducting airways relative to that of lung tissues [3]. Hysteresis is the looping on the transmural pressure-volume relationship, a mechanical characteristic of structures with imperfect elasticity. According to Froeb and Mead [3], bronchodilation will occur after DI if bronchial hysteresis prevails. Airway smooth muscle tone is a major determinant of the effect of a DI and acute bronchoconstriction has been shown to increase bronchial hysteresis [4]. Thus, normal subjects or asthmatics with normal lung function challenged with methacholine, usually bronchodilate, in response to DI [5]. Conversely, asthmatics with spontaneous airway obstruction may show more complex mechanical changes. As a result of various factors such as airway or lung inflammation, airway closure and/ or constriction of small airways, parenchymal hysteresis may prevail over airway hysteresis [1]. These patients therefore frequently demonstrate a bronchoconstrictor response to DI $[1,2]$.

In contrast to the large number of studies on the effects of volume history on lung mechanics in adult subjects [6, 7], little data are available in children. In adults, the effect is usually assessed from successive partial and maximal forced expirations. The skill required to perform this manoeuvre frequently precludes its use in children. An alternative is the forced oscillation technique, which can be used to measure the respiratory resistance $(R \mathrm{rs})$ during tidal breathing. The bronchodilatory effect of DI has thus been demonstrated in children challenged with methacholine $[8,9]$. Symptomatic asthma may be responsible for a different respiratory response to DI, but this hypothesis has received little attention in children. The aim of this study was to describe the effect of a DI on respiratory impedance in asthmatic children with spontaneous AO.

\section{Material and methods}

\section{Patients}

Twenty-one asthmatic children (13 males) aged 5-15 yrs, referred to the laboratory for lung function testing, were included in the study on the basis of a spontaneous AO. The subjects were separated into two groups according to the severity of AO. Group 1 included twelve children with mild AO, i.e. baseline forced expiratory volume in one second (FEV1) was 
$\geqslant 75 \%$ predicted [10]. Five of them were on inhaled steroids. Group 2 included nine children with moderate-to-severe AO, i.e. their baseline FEV1 was $\geqslant 70 \%$ pred. Five subjects were on inhaled steroids. $\beta$-Agonist medications were discontinued $\geqslant 12 \mathrm{~h}$ prior to the measurements. The two groups had similar anthropometric characteristics. Baseline FEV1 and maximum midexpiratory flow (MMEF) were obviously significantly larger in group 1 than in group $2(\mathrm{p}<0.001$, table 1$)$.

\section{Measurement of respiratory impedance}

The apparatus for the measurement of respiratory impedance ( $Z$ rs) (Pulmosfor, SEFAM, Vandoeuvre les Nancy, France) has been described in detail previously [11], except for significant modification of the software for more convenient use and improved handling of data. Briefly, the input pressure was delivered by a loud-speaker around the subject's head enclosed in a canopy (head generator technique). Pressure difference and hence flow across the upper airway wall were thus significantly reduced. An acquisition period lasted 30-50 s during which sinusoidal pressure was applied at $12 \mathrm{~Hz}$. The children wore nose clips and breathed through a mouthpiece connected to a Fleisch no 1 pneumotachograph (Metabo, Hepalinges, Switzerland) attached to a differential pressure transducer (Micro 176PC14HD2, Honeywell $\pm 35 \mathrm{hPa}$; Scarborough, Ontario, Canada). The common mode rejection ratio of the flow channel was $>60 \mathrm{~dB}$ at $12 \mathrm{~Hz}$. The input pressure was measured with an identical transducer, matched to the first within $1 \%$ of amplitude and $2^{\circ}$ of phase $\leqslant 32 \mathrm{~Hz}$. Pressure and flow signals were low-pass filtered at $32 \mathrm{~Hz}$ using analog filters and digitized at a sampling rate of $192 \mathrm{~Hz}$. The breathing component in the signals was eliminated using a 4th order Butterworth high-pass filter with a corner frequency of $6 \mathrm{~Hz}$. The signals were analysed oscillation cycle per oscillation cycle providing 12 measurements per second. Fourier coefficients of pressure and flow at $12 \mathrm{~Hz}$ were computed and combined to obtain $R$ rs and respiratory reactance $(X \mathrm{rs})$. A correction was applied for the $2.1 \mathrm{~ms}$ time constant of the pneumotachograph. Noisy data were finally eliminated by filtering out $Z_{r s}$ values lying outside the $99 \%$ confidence interval, i.e. lower or higher than the mean $\pm 3 \mathrm{sD}$. The procedure was repeated 3 times. After each acquisition, time courses of tidal flow and volume, $R$ rs and $X$ rs were displayed on the computer screen. Recordings showing marked irregular breathing or evidence of glottic closure were discarded.
Measurement of forced expiratory volume in one
second

FEV1 was measured using an electronic flow meter (Masterscope Erich Jaeger GmbH, Würtzburg, Germany). Forced expiratory manoeuvres were repeated up to five times and the flow/volume curve with the highest sum forced vital capacity+FEV1 was retained. In order to avoid change in volume history immediately prior to studying the effect of DI, measurements of FEV1 were, in all instances, obtained following $Z$ rs.

\section{Drug administration}

Reversibility of AO was induced by administering two puffs of salbutamol (Ventoline $\mathbb{R}, 100 \mu \mathrm{g} \cdot \mathrm{puff}^{-1}$ ) through a nonelectrostatic inhalation chamber (NesSpacer, AstraZeneca, Lund, Sweden). The drug delivery system was triggered while the child started to inhale slowly from the chamber. The subject was then asked to hold their breath for $5 \mathrm{~s}$ at end inspiration. Normal breathing was resumed for a few seconds and the second puff was delivered in the same manner. $Z_{\text {rs }}$ and FEV1 measurements were repeated $10 \mathrm{~min}$ thereafter.

\section{Data analysis}

At baseline and after salbutamol, a first acquisition period was obtained. A second acquisition followed immediately, during which the child was asked to take a deep breath. The data were retained when the corresponding volume was at least twice as large as average tidal volume. Three respiratory cycles were analysed before (preDI) and three after DI (postDI). The visual inspection of flow and volume tracings allowed the selection of breathing cycles, usually skipping the first breath immediately after the DI. On both preDI and postDI epochs, mean $R$ rs was calculated in inspiration ( $R$ rsi) and expiration $(R \mathrm{rse})$ as well as mean $X$ rs for the whole respiratory cycle. The magnitude of the Rrs response to DI was expressed by the difference between post and preDI $R$ rsi $(\Delta R \mathrm{rsi})$.

To assess the effects of degree of airway obstruction, DI and bronchodilation, an analysis of variance (ANOVA) design with two repetitions (DI and salbutamol) and one factor (FEV1 $\leqslant 70 \%$ or $\geqslant 75 \%$ ) was used. When a $\mathrm{F}$ ratio indicated a significant effect, a paired t-test was used to locate the difference more precisely. Standard linear regression analysis was used to evaluate the relationship between degree of airway obstruction and change in Rrsi induced by DI.

Table 1.-Characteristics of children with mild (group 1) and moderate-to-severe (group 2) airway obstruction

\begin{tabular}{ccccccc}
\hline Group & Subjects & Sex M:F & Age yrs & Height $\mathrm{m}$ & FEV1\% pred & MMEF \% pred \\
\hline 1 & 12 & $7: 5$ & $9.1 \pm 0.8$ & $1.34 \pm 0.05$ & $85 \pm 1$ & $52 \pm 3$ \\
2 & 9 & $6: 3$ & $8.8 \pm 1.5$ & $1.35 \pm 0.07$ & $58 \pm 5^{* * *}$ & $26 \pm 5^{* * *}$ \\
\hline
\end{tabular}

Data are presented as mean \pm SEM. FEV1: forced expiratory volume in one second; M: male; F: female; MMEF: maximum midexpiratory flow. $* * *: \mathrm{p}<0.01$ versus corresponding value in group 1 . 
A p-value $<0.05$ was used to define statistical significance. Data are expressed as mean \pm SEM.

\section{Results}

ANOVA showed that: 1) Rrsi, $R$ rse and $X$ rs were all significantly altered by salbutamol $(\mathrm{p}<0.002), 2) X \mathrm{rs}$ was significantly different between patient groups $(\mathrm{p}=0.005)$ and 3) significant interaction on Rrsi was demonstrated for patient groups (i.e. degree of AO), DI and salbutamol $(\mathrm{p}<0.04)$.

At baseline, there was no significant difference in $R$ rsi or Rrse between group 1 and 2 (table 2 and 3 ), while $X$ rs was significantly lower in group 2 than in group $1(\mathrm{p}<0.02$, table 4$)$. Patterns of response to DI are illustrated in figures 1 and 2. Recordings were taken from one patient in each group. The DI induced a significant decrease in $R \mathrm{rsi}$ in group $1 \quad \mathrm{p}=0.01$, table 2), while the change in Rrse did not reach statistical significance $(\mathrm{p}=0.08$, table 3$)$. There was no significant change in $X_{\text {rs }}$ (table 4$)$. In group 2, neither $R$ rsi, Rrse nor $X$ rs were significantly different before and after DI (table 2-4). Interestingly, it was also found that, in contrast with the preDI value, postDI Rrsi was significantly lower in group 1 than in group $2(\mathrm{p}=0.05$, table 2$)$.

Table 2.-Respiratory resistance in inspiration (Rrsi) in children with mild (group 1) and moderate-to-severe (group 2) airway obstruction before (pre) and after (post) deep inhalation (DI)

\begin{tabular}{lccc}
\hline Group & $\begin{array}{c}\text { Subjects } \\
\mathrm{n}\end{array}$ & \multicolumn{2}{c}{$R \mathrm{rsi} \mathrm{hPa} \cdot \mathrm{s} \cdot \mathrm{L}^{-1}$} \\
\cline { 3 - 4 } & & Baseline & Salbutamol \\
\hline & 12 & $10.5 \pm 1.2$ & $5.9 \pm 0.6^{* * * *}$ \\
preDI & & $9.0 \pm 0.9^{\#}$ & $5.7 \pm 0.6^{* * *}$ \\
postDI & 9 & $12.5 \pm 1.6$ & $6.8 \pm 1.0^{* * *}$ \\
preDI & & $13.0 \pm 1.9^{*}$ & $7.0 \pm 1.1^{* * *}$ \\
postDI & &
\end{tabular}

Table 3.-Respiratory resistance in expiration (Rrse) in children with mild (group 1) and moderate-to-severe (group 2) airway obstruction before (pre) and after (post) deep inhalation (DI)

\begin{tabular}{lccc}
\hline Group & $\begin{array}{c}\text { Subjects } \\
\mathrm{n}\end{array}$ & \multicolumn{2}{c}{$R \mathrm{rse} \mathrm{hPa} \cdot \mathrm{s} \cdot \mathrm{L}^{-1}$} \\
\cline { 3 - 4 } & & Baseline & Salbutamol \\
\hline 1 & 12 & & \\
preDI & & $12.2 \pm 1.7$ & $7.2 \pm 0.8^{\#}$ \\
postDI & 9 & $10.7 \pm 1.3$ & $7.1 \pm 0.9^{\#}$ \\
2 & & $14.3 \pm 1.8$ & $8.6 \pm 1.2^{\#}$ \\
preDI & & $14.5 \pm 2.1$ & $8.4 \pm 1.2^{\#}$ \\
\hline
\end{tabular}

Data are presented as mean \pm SEM. \#: $\mathrm{p}<0.005$ versus corresponding baseline.
Table 4.-Respiratory reactance ( $X_{\mathrm{rs}}$ ) in children with mild (group 1) and moderate-to-severe (group 2) airway obstruction before (pre) and after (post) deep inhalation (DI)

\begin{tabular}{|c|c|c|c|}
\hline \multirow[t]{2}{*}{ Group } & \multirow{2}{*}{$\begin{array}{c}\text { Subjects } \\
\text { n }\end{array}$} & \multicolumn{2}{|c|}{$X_{\mathrm{rs} \mathrm{hPa}} \cdot \mathrm{s} \cdot \mathrm{L}^{-1}$} \\
\hline & & Baseline & Salbutamol \\
\hline \multirow{3}{*}{$\begin{array}{l}1 \\
\quad \text { preDI } \\
\text { postDI }\end{array}$} & 12 & & \\
\hline & & $0.8 \pm 0.4$ & $1.4 \pm 0.1$ \\
\hline & & $1.1 \pm 0.2$ & $1.5 \pm 0.1$ \\
\hline${ }^{2}$ preDI & 9 & $-1.0 \pm 0.6$ & $0.8 \pm 0.2^{\#, \boldsymbol{\oplus}}$ \\
\hline postDI & & $-0.9 \pm 0.8$ & $0.7 \pm 0.3^{\#, \oplus}$ \\
\hline
\end{tabular}

Data are presented as mean \pm SEM. ${ }^{*}: \mathrm{p}<0.03$ versus corresponding baseline; ${ }^{\uparrow}: \mathrm{p}<0.02$ versus group 1 .
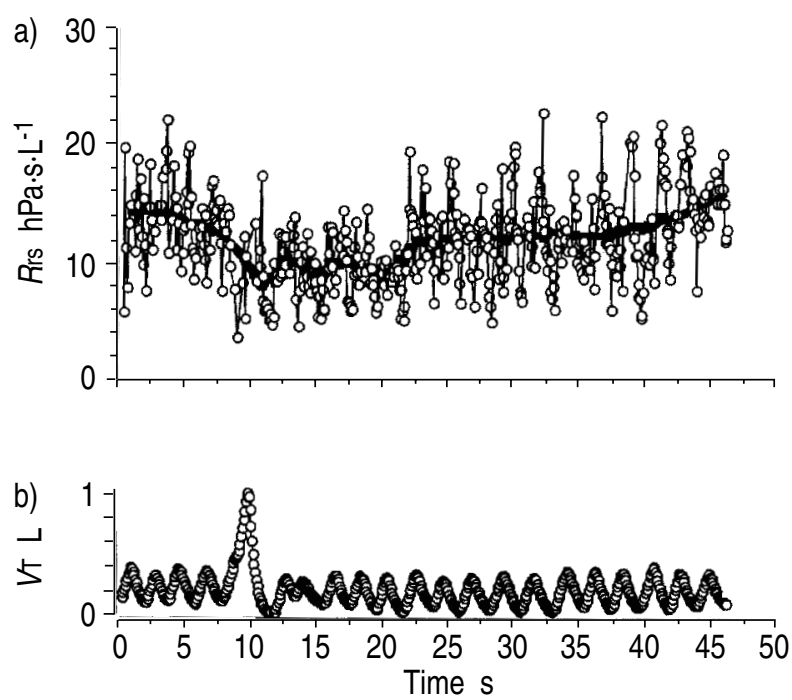

Fig. 1. - Time course of a) respiratory resistance $(R \mathrm{rs})$ and b) tidal volume $(V \mathrm{~T})$ during a deep inhalation (DI) in an asthmatic child with mild airway obstruction (baseline forced expiratory volume in one second $87 \%$ pred). Each dot on the Rrs plot corresponds to one oscillation cycle. Most of the instantaneous variations of $R \mathrm{rs}$ are related to respiratory flow. The continuous line corresponds to the smoothed data and shows that DI is associated with a transient drop in $R$ rs which then returns progressively back to baseline within $30 \mathrm{~s}$.

$\Delta R$ rsi was found to be significantly different between groups i.e. negative in group $1\left(-1.5 \pm 0.5 \mathrm{hPa} \cdot \mathrm{s} \cdot \mathrm{L}^{-1}\right)$ but not in group $2\left(0.5 \pm 0.5 \mathrm{hPa} \cdot \mathrm{s} \cdot \mathrm{L}^{-1}, \mathrm{p}=0.02\right)$. Studying the relationship between the degree of AO and the response to DI during AO disclosed a significant correlation between FEV1 \% pred and $\Delta R$ rsi ( $\mathrm{p}=0.02$, fig 3). A similar relationship was found between $\Delta R$ rsi and MMEF \% pred $(\mathrm{p}=0.06)$, but this did not reach statistical significance.

After salbutamol, FEV1 increased significantly, from $1.6 \pm 0.1 \mathrm{~L}$ to $1.9 \pm 0.2 \mathrm{~L}$ in group $1(\mathrm{p}<0.001)$ and from $1.1 \pm 0.2 \mathrm{~L}$ to $1.6 \pm 0.3 \mathrm{~L}$ in group $2(\mathrm{p}<$ $0.004)$. In both groups, Rrsi and Rrse decreased significantly $(\mathrm{p}<0.005)$, while $X$ rs did not change in group $1(\mathrm{p}=0.1)$ and increased in group $2(\mathrm{p}=0.03)$ 

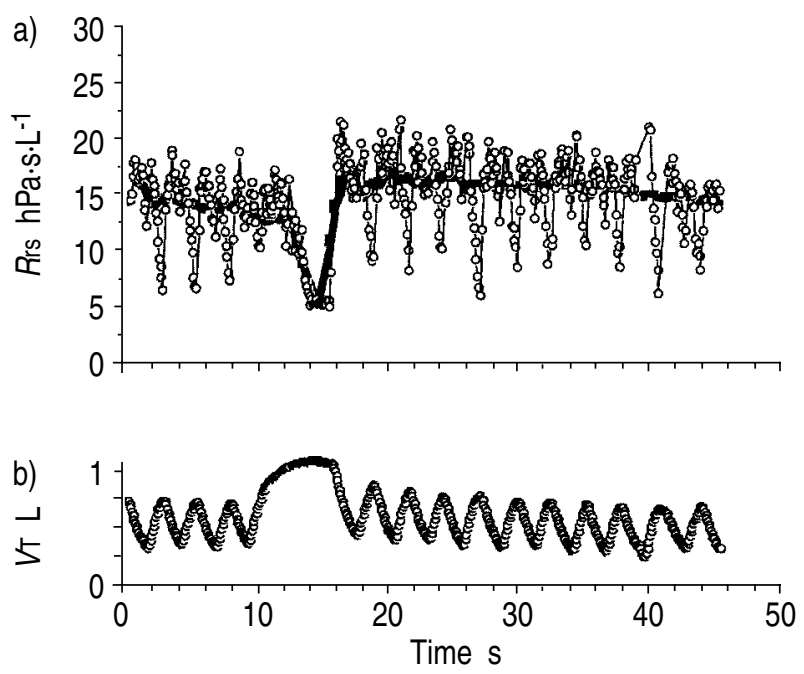

Fig. 2. - Time course of a) respiratory resistance $(R \mathrm{rs})$ and b) tidal volume $(V \mathrm{~T})$ during a deep inhalation (DI) in an asthmatic child with severe airway obstruction (baseline forced expiratory volume in one second $34 \%$ pred). The deep inhalation is followed by an increase in $R$ rs which then decreases progressively back toward baseline.

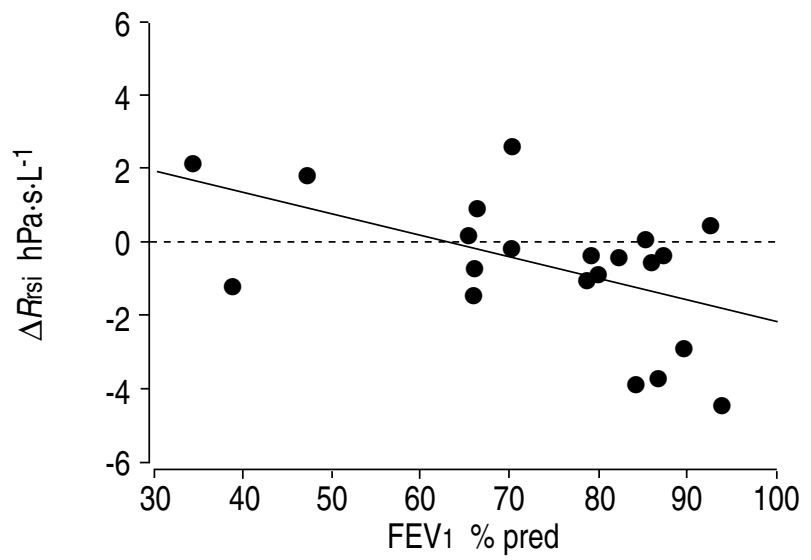

Fig. 3.-The magnitude of the change in inspiratory resistance (Rrsi) induced by deep inhalation shows significant correlation to the degree of baseline airway obstruction expressed as forced expiratory volume in one second $\left(F E V_{1}\right) \%$ pred $(\mathrm{p}=0.02)$. Negative values of $\Delta R$ rsi indicate bronchodilation.

(tables 2-4). Neither Rrsi nor Rrse were found to be different between groups (table 2 and 3 ), but $X$ rs was still lower in group 2 than in group 1 after salbutamol $(\mathrm{p}=0.02$, table 4$)$. DI induced no significant change in any parameter in either group (table 2-4).

\section{Discussion}

To summarize, in this population of young asthmatics the response pattern to DI appears to be dependent on the severity of spontaneous AO. Children with mild AO show a significant decrease in Rrsi after DI while those with moderate-to-severe AO show either no improvement or worsening.

\section{Methodological issues}

Comparing flows resulting from consecutive partial and maximal forced expiratory manoeuvres is viewed as the gold standard for studying airway mechanics in relation to volume history [6,7]. Measurement of $Z$ rs does not require active cooperation and is therefore particularly adapted to children. Relatively high excitation frequencies are necessary because of the harmonic content of respiratory flow in children and, in the authors' experience, reliable data are difficult to obtain $<10 \mathrm{~Hz}$. A study of $\mathrm{Zrs}$ using multiple frequency input signals in a normal population of children showed that a coherence function $\geqslant 0.95$ could be obtained in all cases $\geqslant 12 \mathrm{~Hz}$ [12]. The drawback of high-frequency pressure oscillations is the potential source of error related to the proximal shunting of airway flow, which may be particularly significant in children with AO [13, 14]. Upper airway wall motion was minimized in this study by varying transrespiratory pressure around the subject's head thereby reducing the pressure difference across the cheeks [15].

The analysis of maximal/partial forced expiratory flows allows a single-breath evaluation of the effects of DI, while measurements of $Z$ rs must be performed over a number of breaths to provide meaningfully interpretable data. Because the bronchodilatory effect of DI has been reported to subside with a circa $11 \mathrm{~s}$ time constant in adults [16], the analysis was limited to three breaths taken shortly after the DI in order to minimize the effect of time. The relationship between $\triangle \mathrm{Rrsi}$ and FEV1 in children (fig. 3) was consistent with previous reports on maximal/partial expiratory flow manoeuvres in adults where the magnitude of the bronchodilator effect of DI was inversely related to the severity of spontaneous AO [2]. Thus the authors believe that the measuring conditions in this study were optimal for detecting respiratory mechanical events directly associated with the DI.

\section{Lung function in groups 1 and 2}

It may be surprising that in two groups of patients with different FEV1, baseline Rrs was not significantly different. In both groups, there was a marked fall in $R$ rs after salbutamol, indicating that baseline values were clearly abnormal. Forced expiration and tidal breathing are two different patterns during which findings relative to AO may not be parallel. During a forced expiratory manoeuvre, and in contrast to tidal breathing, reflex abduction of the larynx minimizes the contribution of the upper airways [17] and decreased FEV1 is usually taken as an indication of flow limitation in the intrathoracic airways. This may not necessarily reflect an increase in the resistance of the whole airway tree, to which the pharynx and larynx contribute significantly, with subsequent effect on $R$ rs. Nevertheless, it is worth noting that after DI a significant difference in baseline Rrs was observed between the 2 groups. There are further indications, in keeping with the lower FEV1, that abnormalities of 
intrathoracic airways were more significant in group 2 than in group 1 . $X$ rs was clearly abnormal in the group with severe AO with reference to normative values in children (about $+1 \mathrm{hPa} \cdot \mathrm{s} \cdot \mathrm{L}^{-1}$ at $12 \mathrm{~Hz}$ ) [12] and was also significantly lower than in group 1 . The negative $X$ rs may reflect increased apparent respiratory elastance due, for instance, to nonhomogeneous behaviour of the respiratory system [18] and was significantly increased from baseline after salbutamol. In group $1, X$ rs was positive, expressing dominant airway inertance at that frequency and was unaffected by salbutamol. As a result, $X$ rs was still significantly lower in group 2 than in group 1 after bronchodilator inhalation. Therefore, the findings in group 2 are consistent with moderate-to-severe AO associated with an increase in apparent respiratory elastance being partly reversed by the bronchodilator.

\section{Effects of deep inhalation}

The decrease in Rrs after DI in group 1 was found to be significant in inspiration but not in expiration. It has been shown in humans that during tidal expiration, progressive glottis closure usually occurs in parallel with the decrease in volume [19]. Closure of the glottis associated with bronchoconstriction [20-22] is also more prominent in expiration than in inspiration [22]. Furthermore, evidence has been provided that laryngeal resistance may be altered by DI, the direction of the change depending on the presence of an AO. After DI, laryngeal resistance was decreased in normal subjects and stable asthmatics, and increased in normal subjects with methacholine induced bronchoconstriction as well as in asthmatics during asthma attack [23]. Such an increase in laryngeal resistance in response to DI would be more effective in increasing $R \mathrm{rs}$ in expiration than in inspiration, thus minimizing the expression in $R$ rse of the bronchodilatory effect of DI.

Results obtained from group 1 are consistent with the study by MilanESE et al. [8], where spontaneous AO was suspected in nine preschool children on the basis of a reduction in Rrs $>30 \%$ after salbutamol. Milanese et al. [8] showed significant decrease in $R$ rs after DI and no change in resonant frequency at baseline. Although FEV1 was not measured, the response resembles that observed in subjects from group 1 of the present study, where Rrsi was significantly decreased after DI but $X$ rs was unchanged. However, the data on salbutamol differ between the two studies, in that Milanese et al. [8] described a significant increase in $R$ rs after DI, while no significant variation was found in the present study (tables 2-4). It should be noted that the characteristics of the two populations were different. The former study included only preschool children while the present study included both preschool and school-age children. This may be of particular relevance since a recent report on the bronchodilator response to DI in asthmatics with varying degrees of AO described a significant inverse relationship between the magnitude of this response and age [24]. In addition, MiLANESE et al. [8] studied children with recurrent wheezing, requiring only short acting $\beta$-agonists on an as-necessary basis [8], while some of the asthmatic children in the present study required inhaled steroids and/or presented with significant underlying respiratory disease. In this regard, it is worth noting that, after salbutamol, $X$ rs was still significantly lower in group 2 than in group 1 .

In group 2, data are clearly different from prior observations in children where AO was acutely induced by methacholine inhalation $[8,9]$ and where both the increase in $R$ rs and the decrease in $X$ rs were found to be significantly reversed by DI [9]. The interpretation was that, in the context of an acute bronchoconstriction, airway hysteresis was likely to be increased and DI would promote bronchodilation $[3,6,7]$. Conversely, the lack of increase in $R$ rs and decrease in $X$ rs in subjects in the present study may indicate that the effect of DI on airway hysteresis was counteracted by an increase in parenchymal hysteresis. Lung hyperinflation is a common finding of chronic asthma [25]. It may be important in determining the response to DI, since it may be associated with increased hysteresis of lung tissues which favours a bronchoconstrictor response to DI. The present study lacked a systematic estimation of total lung capacity to evaluate the contribution of this mechanism. It is also possible that constriction taking place distal to the respiratory bronchioles could induce an increase in parenchymal hysteresis [1]. Another possible explanation is that inflammation and oedema within the airway wall decreases elastic load on intraparenchymatous airways leading to less interdependence between these airways and lung tissues [26]. However, figure 2, which indicates bronchoconstriction after DI, does not favour this hypothesis since a significant decrease in $R$ rs occurs during the inspiratory manoeuvre, suggesting some degree of interdependence between lung parenchyma and conducting airways.

In conclusion, in this population of asthmatic children, the bronchomotor effect of deep inhalation may be demonstrated by measuring respiratory resistance with the forced oscillation technique. Bronchodilation appears to be more readily detected in inspiration than in expiration in subjects with mild airway obstruction, but not in those with moderateto-severe airway obstruction. The magnitude of change in respiratory resistance in inspiration induced by deep inhalation is inversely related to the degree of baseline airway obstruction. Deep inhalation has no effect on respiratory reactance which nonetheless appears to differentiate mild airway obstruction from moderate-to-severe airway obstruction and to be readily sensitive to salbutamol in the latter group. Further studies are needed to assess the usefulness of the respiratory resistance response to deep inhalation in characterising airway disease in children.

Acknowledgements. The authors are grateful to B. Chalon, C. Choné, G. Colin and S. Méline for technical assistance, N. Bertin and C. Creusat for secretarial help and R. Peslin for fruitful discussion. 


\section{References}

1. Burns CB, Taylor WR, Ingram RH. Effects of deep inhalation in asthma: relative airway and parenchymal hysteresis. J Appl Physiol 1985; 59: 1590-1596.

2. Lim TK, Pride NB, Ingram RH. Effects of volume history during spontaneous and acutely induced airflow obstruction in asthma. Am Rev Respir Dis 1987; 135: 591-596.

3. Froeb HF, Mead J. Relative hysteresis of the dead space and lung in vivo. J Appl Physiol 1968; 25: 244-248.

4. Sasaki H, Hoppin FG. Hysteresis of contracted airway smooth muscle. J Appl Physiol 1979; 47: 1251-1262.

5. Fish JE, Ankin MG, Kelly JF, Peterman VI. Regulation of bronchomotor tone by lung inflation in asthmatic and nonasthmatic subjects. J Appl Physiol 1981; 50: 1079-1086.

6. Ingram RH. Relationship among airway-parenchymal interactions, lung responsiveness, and inflammation in asthma. Chest 1995; 107: 148s-152s.

7. Pellegrino R, Sterk PJ, Sont JK, Brusasco V. Assessing the effect of deep inhalation on airway calibre: a novel approach to lung function in bronchial asthma and COPD. Eur Respir J 1998; 12: 1219-1227.

8. Milanese M, Mondino C, Tosca M, Canonica GW, Brusasco V. Modulation of airway caliber by deep inhalation in children. J Appl Physiol 2000; 88: 12591264.

9. Marchal F, Schweitzer C, Moreau-Colson C. Respiratory impedance response to a deep inhalation in children. Pediatr Pulmonol 2002 (in press).

10. Knudson RJ, Lebowitz MD, Holberg CJ, Burrows B. Changes in the normal maximal expiratory flowvolume curve with growth and aging. Am Rev Respir Dis 1983; 127: 725-734.

11. Peslin R, Marchal F, Duvivier C, Ying Y, Gallina C. Evaluation of a modified head generator for respiratory impedance measurement. Eur Respir Rev 1991; 1: 140-145.

12. Mazurek H, Willim G, Marchal F, Haluszka J, Tomalak W. Input respiratory impedance measured by head generator in preschool children. Pediatr Pulmonol 2000; 30: 47-55.

13. Cauberghs M, van de Woestijne KP. Effect of upper airway shunt and series properties on respiratory impedance measurements. J Appl Physiol 1989; 66: 2274-2279.
14. Marchal F, Mazurek H, Habib M, Duvivier C, Derelle $\mathrm{J}$, Peslin R. Input respiratory impedance to estimate airway hyperreactivity in children: standard method versus head generator. Eur Respir J 1994; 7: 601-607.

15. Peslin R, Duvivier C, Didelon J, Gallina C. Respiratory impedance measured with head generator to minimize upper airway shunt. J Appl Physiol 1985; 59: 1790-1795.

16. Parham WM, Shepard RH, Norman PS, Fish JE. Analysis of time course and magnitude of lung inflation effects on airway tone: relation to airway reactivity. Am Rev Respir Crit Care Med 1983; 128: 240-245.

17. Clément J, Stanescu DC, van de Woestijne KP. Glottis opening and effort-dependent part of the isovolume pressure-flow curves. J Appl Physiol 1973; 34: 18-22.

18. Peslin R, Fredberg JJ. Oscillation mechanics of the respiratory system. In: Fishman PA, Macklem PT, Mead J, eds. Handbook of Physiology. The respiratory system. Mechanics of breathing. Baltimore, William \& Wilkins, 1986; pp. 145-177.

19. Brancatisano T, Dodd D, Engel LA. Factors influencing glottic dimensions during forced expiration. $J$ Appl Physiol 1983; 55: 1825-1829.

20. England SJ, Ho V, Zamel N. Laryngeal constriction in normal humans during experimentally induced bronchoconstriction. J Appl Physiol 1985; 58: 352-356.

21. Higgenbottam T. Narrowing of glottis opening in humans associated with experimentally induced bronchoconstriction. J Appl Physiol 1980; 49: 403-407.

22. Collett PW, Brancatisano T, Engel LA. Changes in the glottic aperture during bronchial asthma. Am Rev Respir Dis 1983; 128: 719-723.

23. Sekizawa K, Yanal M, Sasaki H, Takishima T. Effect of a previous voluntary deep breath on laryngeal resistance in normal and asthmatic subjects. $J$ Appl Physiol 1987; 63: 1406-1412.

24. Pacini F, Filippelli M, Duranti R, et al. Reduction in bronchodilation following a deep inhalation is poorly related to airway inflammation in asthma. Eur Respir J 1999; 14: 1055-1060.

25. Pellegrino R, Brusasco V. On the causes of lung hyperinflation during bronchoconstriction. Eur Respir $J$ 1997; 10: 468-475.

26. Macklem PT. A theoretical analysis of the effect of airway smooth muscle load on airway narrowing. $\mathrm{Am}$ J Respir Crit Care Med 1996; 153: 83-89. 